\title{
EDITORIAL
}

Jeff D. Eldredge · Rajat Mittal

\section{Recent developments in multiphysics computational models of physiological flows}

Published online: 30 January 2016

(C) Springer-Verlag Berlin Heidelberg 2016

\begin{abstract}
A mini-symposium on computational modeling of fluid-structure interactions and other multiphysics in physiological flows was held at the 11th World Congress on Computational Mechanics in July 2014 in Barcelona, Spain. This special issue of Theoretical and Computational Fluid Dynamics contains papers from among the participants of the mini-symposium. The present paper provides an overview of the mini-symposium and the special issue.
\end{abstract}

\section{Overview of the mini-symposium}

Capabilities for numerical simulations of physiological flows have evolved tremendously in the last decade. This evolution is primarily due to notable advances in computational architecture, numerical methodologies, modeling of the rheology and elastic characteristics of biological materials, and pathways from medical imaging to flow computation. Within the advancement of numerical methodologies, there are two fronts on which developments have been particularly notable. One of these fronts involves strategies for the immersion of complex geometries in simple static grids. These so-called immersed boundary methods, which were first introduced in the 1970s but have seen a resurgence in the last decade, have gone far to address the challenges of simulation with complex moving boundaries, which are particularly troublesome in physiology. On the other front, researchers are devising new methods for coupling the fluid dynamics with other relevant physics: the structural dynamics of soft tissues, the electrophysiology of the heart muscles, and the biochemistry of fluid components, such as thrombi or aerosol particulates. The developments on these fronts have opened a wide new world for computational mechanics. Many of these advances have been fueled by productive partnerships that have emerged between engineers, physicians, and biomedical researchers, to the point that simulations are even starting to provide guidance on patient-specific diagnosis and surgical planning.

The authors of this introductory paper organized a mini-symposium on the topic of multiphysical modeling of physiological flows at the 11th World Congress on Computational Mechanics, held in Barcelona in July 2014, in which the results of several such partnerships were presented over the course of two sessions. The mini-symposium brought together several researchers who are making novel contributions to the computation of flows and associated multiphysics problems in a variety of physiological contexts. The twofold objective of the mini-symposium was to highlight the state of the art in modeling of physiological flows and to provide

J. D. Eldredge $(\varangle)$

Mechanical and Aerospace Engineering, University of California, Los Angeles, 420 Westwood Plaza, Los Angeles, CA 90095-1597, USA

E-mail: eldredge@seas.ucla.edu

R. Mittal

Department of Mechanical Engineering, Johns Hopkins University, 3400 N. Charles Street, Baltimore, MD 21218, USA

E-mail: mittal@jhu.edu 
a forum for the comparative assessment of the underlying numerical methodologies that are making these applications possible.

\section{The papers}

For this special issue of Theoretical and Computational Fluid Dynamics, each of the speakers in the Barcelona mini-symposium was invited to contribute a paper, either on the topic they presented or on other relevant work. The focus of this special issue is the same as that of the original mini-symposium: applications and numerical techniques of the mechanics of physiological flows. Ultimately, eight papers (seven from among the mini-symposium participants and one additional paper) were accepted for this special issue. All except for two papers address issues in cardiovascular hemodynamics, and this reflects, to some degree, the large inroads that fluid mechanicians have made into the field of cardiology. Among the six papers in the area of cardiovascular hemodynamics, topics range from the hemodynamics of natural [4,8] and prosthetic valves [1], to fundamental investigations of normal [3,9] and diseased states [7] of the cardiovascular system. The paper of Dillard et al. [2] describes a new method for going from videos to CFD ready models and this method is applicable to a wide variety of biological systems. Finally, the paper of Huang et al. [5,6] addresses obstructive sleep apnea, a prevalent disease condition that is being increasingly targeted in modern medicine.

\section{References}

1. De Vita, F., de Tullio, M.D., Verzicco, R.: Numerical simulation of the non-newtonian blood flow through a mechanical aortic valve: Non-Newtonian blood flow in the aortic root. Theor. Comput. Fluid Dyn. 30(1-2) (2016). doi:10.1007/s00162-0150369-2

2. Dillard, S.I., Buchholz, J.H.J., Udaykumar, H.S.: From video to computation of biological fluid-structure interaction problems. Theor. Comput. Fluid Dyn. 30(1-2) (2016). doi:10.1007/s00162-015-0385-5

3. Flamini, V., DeAnda, A., Griffith, B.E.: Immersed boundary-finite element model of fluid-structure interaction in the aortic root. Theor. Comput. Fluid Dyn. 30(1-2) (2016). doi:10.1007/s00162-015-0374-5

4. Gilmanov, A., Sotiropoulos, F.: Comparative hemodynamics in an aorta with bicuspid and trileaflet valves. Theor. Comput. Fluid Dyn. 30(1-2) (2016). doi:10.1007/s00162-015-0364-7

5. Huang, C.J., Huang, S.C., White, S.M., Mallya, S.M., Eldredge, J.D.: Erratum to: Toward numerical simulations of fluidstructure interactions for investigation of obstructive sleep apnea. Theor. Comput. Fluid Dyn. 30(1-2) (2016). doi:10.1007/ s00162-015-0372-7

6. Huang, C.J., Huang, S.C., White, S.M., Mallya, S.M., Eldredge, J.D.: Toward numerical simulations of fluid-structure interactions for investigation of obstructive sleep apnea. Theor. Comput. Fluid Dyn. 30(1-2) (2016). doi:10.1007/s00162-0150368-3

7. Mukherjee, D., Padilla, J., Shadden, S.C.: Numerical investigation of fluid-particle interactions for embolic stroke. Theor. Comput. Fluid Dyn. 30(1-2) (2016). doi:10.1007/s00162-015-0359-4

8. Traeger, B., Srivatsa, S.S., Beussman, K.M., Wang, Y., Suzen, Y.B., Rybicki, F.J., Mazur, W., Miszalski-Jamka, T.: Methodological inaccuracies in clinical aortic valve severity modeling of ct-derived aortic valve anatomy. Theor. Comput. Fluid Dyn. 30(1-2) (2016). doi:10.1007/s00162-015-0370-9

9. Vedula, V., Seo, J.H., Lardo, A.C., Mittal, R.: Effect of trabeculae and papillary muscles on the hemodynamics of the left ventricle. Theor. Comput. Fluid Dyn. 30(1-2) (2016). doi:10.1007/s00162-015-0349-6 